Cahiers $d u$ MONDE RUSSE

\section{Cahiers du monde russe}

Russie - Empire russe - Union soviétique et États indépendants

$53 / 4 \mid 2012$

Varia

\title{
Svetlana Gorshenina, Asie Centrale
}

\section{Beatrice Pénati}

URL : http://journals.openedition.org/monderusse/7754

DOI : $10.4000 /$ monderusse. 7754

ISSN : $1777-5388$

Éditeur

Éditions de l'EHESS

Édition imprimée

Date de publication : 15 décembre 2012

ISSN : $1252-6576$

\section{Référence électronique}

Beatrice Pénati, «Svetlana Gorshenina, Asie Centrale », Cahiers du monde russe [En ligne], 53/4 | 2012, mis en ligne le 19 mars 2013, Consulté le 30 avril 2019. URL : http://journals.openedition.org/ monderusse/7754; DOI : 10.4000/monderusse.7754

Ce document a été généré automatiquement le 30 avril 2019

(c) École des hautes études en sciences sociales 


\title{
Svetlana Gorshenina, Asie Centrale
}

\author{
Beatrice Pénati
}

\section{RÉFÉRENCE}

Svetlana GoRSHENINA, Asie Centrale. L'invention des frontières et l'héritage russosoviétique. Paris : CNRS Editions, 2012 (coll. Espaces et milieux), 381 p.

1 Le nouveau livre de la chercheuse franco-ouzbèque Svetlana Gorshenina est issu de son travail de thèse et fait partie d'une trilogie, dont deux autres volumes à paraître porteront sur «l'histoire du concept d'Asie centrale» et sur la cartographie de cette région du monde (p.23). Cet ouvrage, auquel le titre Asie Centrale va assurer un bon référencement dans tout catalogue, se situe au croisement entre histoire, area studies, et géographie. La thèse principale de l'ouvrage - la nature fictive et historiquement déterminée de toute "frontière naturelle »- est bien connue des chercheurs de cette dernière discipline notamment à travers les travaux de Claude Raffestin, que S. Gorshenina ne manque pas de mentionner. L'auteur démontre pourquoi et comment cette thèse sied à l'histoire et à la situation présente des frontières extérieures de l'Empire russe en Asie Centrale, voire à ses démarcations internes, y compris aux frontières qui, depuis 1991, définissent le territoire des républiques indépendantes. L'exposition s'organise selon un plan chronologique : la première partie couvre la période antérieure à 1917 ; la seconde porte sur le découpage administratif à l'époque soviétique et se termine par une esquisse des problèmes associés aux frontières depuis 1991. À l'exception du dernier chapitre, S. Gorshenina ne s'intéresse pas à l'histoire locale le long des frontières : elle met l'accent sur la mise en place de ces dernières, plus que sur la vie des habitants qui les côtoient ou les traversent.

2 Les trois premiers chapitres portent respectivement sur «les projets russes de progression vers l'Asie », sur l'occupation de Kuldža (Xinjiang) par les Russes entre 1871 et 1881 , et sur les aspects techniques, diplomatiques, et idéologiques de la définition des frontières externes de l'Empire. Gorshenina adopte le point de vue de l'histoire des idées pour analyser les raisons de la conquête de l'Asie Centrale dans le discours des militaires 
et politiciens russes et étrangers. Elle nous offre une vision synthétique des étapes de l'avancée russe jusqu'à la chute du khanat de Kokand, mais les débats qu'elle évoque se réfèrent plutôt à cette dernière phase qu'à la décision, pourtant cruciale, de quitter la ligne fortifiée d'Orenburg pour s'enfoncer dans la terra incognita des steppes kazakhes. Gorshenina accorde beaucoup plus d'attention aux frontières russes avec les khanats et l'émirat de Buhara, avec la Perse, l'Afghanistan, ou la Chine, qu'aux relations entre nomades et Russes. Cette approche se poursuit dans le troisième chapitre où l'auteur démontre la flexibilité de la notion de "frontière naturelle " avec ses prétentions à la scientificité, du côté russe comme du côté de ceux qui regardaient cette avancée du point de vue de l'Inde britannique. Le mérite principal de cette étude est de distinguer nettement les rêves idéologiques ou les grands projets stratégiques des décisions militaires.

3 Le deuxième chapitre du livre, portant sur la conquête de Kuldža compte parmi les apports les plus originaux de l'ouvrage et pourrait faire l'objet d'une publication particulière. Fondant son étude sur un pan inédit du «fonds Serebrennikov » (auteur de l'ouvrage «classique» (1914) sur la pénétration russe en Asie Centrale), Gorshenina présente de nouvelles données pour discuter le poids des initiatives individuelles dans l'aventure coloniale russe. Bien que l'exemple de Kuldža serve bien la thèse des choix opérés par les personnes et de leur importance, les éléments empiriques que Gorshenina mentionne à propos d'autres épisodes (par exemple, la prise de Taškent) nous semblent moins convaincants.

4 En abordant l'époque soviétique, l'auteur a consciemment pris le risque de répéter ce qui a déjà été écrit sur le thème de la « délimitation nationale ». Bien que le contenu de ces chapitres ne soit pas tout à fait nouveau pour les spécialistes, il demeure néanmoins très utile que le public francophone dispose désormais d'une présentation générale de la question, afin de tordre le cou une fois pour toutes au mythe d'un «Stalin usant de son grand crayon ", du divide et impera, et de la passivité des élites locales. Ici, Gorshenina comble un vide important. Enfin, ce livre se distingue d'autres travaux pour son attention, d'une part, à la relation entre "délimitation nationale» et découpage intérieur, d'autre part, aux ajustements et à la révision des frontières entre 1924 et 1936, notamment via le travail de la commission Magidovič́1. Ce sont des données nouvelles que les historiens de l'Asie centrale soviétique sauront apprécier, bien qu'elles eussent pu être développées davantage.

5 Ceci dit, l'ouvrage n'est pas sans limites. D'abord, il semble que l'auteur n'ait pas suffisamment réfléchi quant à son audience. Les spécialistes y trouveront trop de passages fondés sur une littérature secondaire désormais connue. Cependant, ce livre ne peut avoir valeur de manuel, pour les étudiants ou le grand public, qui y trouveront des renvois trop détaillés ou, au contraire, des références un peu obscures. Par exemple, qui sont «les Djadids» (p. 209)? Qui sont "les dirigeants kazakhs» et «l'élite politique kirghize » (p. 222) ? Qui est l'Asfendiarov nommé à la page 226 ? Étant donné l'importance de ce dernier dans l'histoire de la république turkestanaise, le lecteur-étudiant aurait pu bénéficier d'une note biographique moins laconique que celle proposée dans l'index (pourtant utile).

6 Deuxièmement, on ne trouve pas dans le livre de paragraphes où, de façon systématique ou épisodique, l'auteur explique pourquoi son étude se fonde sur certaines sources et pas d'autres, et quelles en sont leurs limites. Autrement dit, l'historien surtout éprouvera le manque d'une vraie critique des documents. Prenons le cas du «fonds Serebrennikov» 
des archives d'État d'Ouzbékistan, sur lequel Gorshenina bâtit sa reconstruction de la conquête : les matériaux qu'il contient sont-ils vraiment suffisants? Ne correspondent-ils pas à un agenda politico-militaire spécifique qui aurait conduit le compilateur à sélectionner certains papiers et à en ignorer d'autres? La thèse de l'importance des initiatives individuelles dans l'avancée militaire russe dépend-elle de ce choix documentaire, ou serait-elle plausible sur la base d'autres documents ? La même remarque vaut pour les mémoires et les travaux qui, écrits par des contemporains, mais contenant néanmoins des évaluations ex post du processus de conquête, tels par exemple ceux de Snesarev, Miljutin, Kuropatkin, ou Venjukov. De même, l'auteur utilise très naïvement une citation (de seconde main) de Mustafa Čokaev (p.126), alors exilé en Europe, pour décrire le programme politique de l'Autonomie de Kokand. En outre, Gorshenina ne nous dit jamais quelle est la valeur ajoutée des longues citations de travaux d'auteurs européens (par exemple, Leroy-Beaulieu). En ce qui concerne spécifiquement l'usage des sources d'archives, on se demande pourquoi l'auteur limite son attention aux documents accessibles à Taškent et n'utilise ceux conservés à SaintPétersbourg et à Moscou qu'à travers quelques recueils publiés. Ce choix est peut-être justifié, mais il aurait fallu en expliciter les raisons.

7 Troisièmement, le livre de Gorshenina contient une série d'imprécisions qui auraient pu (et dû) être identifiées et corrigées durant le processus de peer review. Certaines d'entre elles sont peut-être le produit d'une simple distraction, par exemple les incohérences et les erreurs dans les translittérations, en dépit de la méticuleuse explication des signes diacritiques dans les premières pages. L'inattention peut aussi expliquer la façon erronée (ou, au mieux, très ambigüe) dont Gorshenina utilise une citation de Curzon (p. 63) : l'auteur britannique y exclut que l'avancée russe ressemble à celle, inexorable, d'un glacier, et en souligne au contraire ses incertitudes et son caractère approximatif. Enfin Gorshenina traduit le mot ziarat par "édifice fortifié »(p.160), alors qu'il signifie « pèlerinage » et ziaratgah « lieu de pèlerinage ».

8 D'autres erreurs sont plus substantielles et un lecteur peu avisé risquerait de reproduire quelques-unes des idées reçues que A.J. Frank et d'autres ont pourtant réfutées. Par exemple, Gorshenina reprend sans aucune discussion le mythe des «nomades faiblement islamisés » (p. 210) et, à la même ligne, simplifie brutalement la distinction faite par T. Uyama entre intellectuels kazakhs « des steppes » et « du sud et de l'ouest », ici décrit un peu hâtivement comme «moins islamisés » et " plus islamisés » (p. 193). Sur le terrain moins glissant de la politique tsariste, Gorshenina est très ambiguë quand elle esquisse la relation entre migration paysanne et avancée en Asie centrale (qualifiée de " colonisation foudroyante », p. 133) : à la lire, il semble que les «offensives militaires» et les accords «signés par les faibles États du Turkestan » soient une conséquence de la migration des paysans russes et du choix du gouvernement impérial de "protéger ses sujets » (p. 41). Or, Gorshenina gomme la distinction entre expansion dans les steppes et dans les oasis, et n'écrit pas clairement qu'au Turkestan la conquête militaire ne suit pas, mais précède, l'arrivée des colons: ce sont bien les «Kirghizes» (Kazakhs) que les Russes disent protéger contre Hiva et Kokand (p. 52-53).

9 En conclusion, l'ouvrage de Svetlana Gorshenina apporte au lecteur spécialiste de nouvelles données empiriques sur des épisodes auparavant négligés, y compris dans l'histoire de la délimitation nationale. En outre, elle parvient à souder les éléments les mieux connus dans un même récit général allant du XIX ${ }^{e}$ au XxI ${ }^{e}$ siècle, au diapason des récents efforts pour reconsidérer le tournant de 1917. Malgré la nécessité d'intégrer et de 
confronter cette lecture avec celle d'autres travaux parus ces vingt dernières années, le public non-spécialiste trouvera dans ce livre une exposition généralement claire, structurée autour d'une thèse plausible, et écrite de façon captivante, d'une série d'événements dont le portrait dans la presse et les ouvrages à grande diffusion reste encore très fautif.

\section{NOTES}

1. I.P. Magidovič, géographe, était le chef de la « Commission pour le découpage administratif de l'Asie centrale », officiellement présidée par I.S. Šelehes (p. 220). 